\title{
A NOVEL APPROACH TO CAMERA CALIBRATION METHOD FOR SMART PHONES UNDER ROAD ENVIRONMENT
}

\author{
Bijun Lee ${ }^{\mathrm{a}}$, Jian Zhou ${ }^{\mathrm{a}}$, Maosheng Ye ${ }^{\mathrm{b}}$,Yuan Guo ${ }^{\mathrm{a}}$

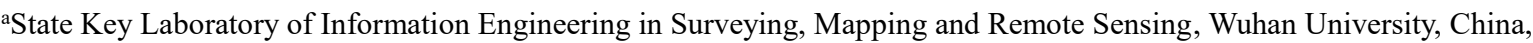 \\ (Lee, zj2007,guoyuan)@whu.edu.cn \\ bSchool of Geodesy and Geomatics, Wuhan University, China, heryms@163.com
}

\section{Commission V, WG V/1}

KEY WORD: Road Lane Markers, Machine Learning, Camera Calibration, Smart Phone

\begin{abstract}
:
Monocular vision-based lane departure warning system has been increasingly used in advanced driver assistance systems (ADAS). By the use of the lane mark detection and identification, we proposed an automatic and efficient camera calibration method for smart phones. At first, we can detect the lane marker feature in a perspective space and calculate edges of lane markers in image sequences. Second, because of the width of lane marker and road lane is fixed under the standard structural road environment, we can automatically build a transformation matrix between perspective space and 3D space and get a local map in vehicle coordinate system. In order to verify the validity of this method, we installed a smart phone in the 'Tuzhi' self-driving car of Wuhan University and recorded more than $100 \mathrm{~km}$ image data on the road in Wuhan. According to the result, we can calculate the positions of lane markers which are accurate enough for the self-driving car to run smoothly on the road.
\end{abstract}

\section{INTRODUCTION}

Camera calibration is the crucial step in photogrammetry, which is the determination of parameters necessary to establish the projection equations between the world coordinate system and image geometry ${ }^{[1]-[2]}$.In self-driving car, obtaining the camera parameters called Pos information is the necessary part for the $3 \mathrm{D}$ reconstruction which is a great implement in the advanced driver assistance systems ${ }^{[3]-[4]}$

There are many approaches for camera calibration. The commonest way is to make full use of the control points in the world coordinate system and their corresponding image points in the image plane to solve the parameters by the collinearity equation or direct linear transformation, namely spatial resection or DLT ${ }^{[5]-[6]}$. Another typical one based on monocular vision, is to use the geometrical relationship of the three vanishing points to calculate the parameters called camera self- calibration ${ }^{[7][8][9]}$. However, both methods mentioned above are not practical and applicable in self-driving car due to their complexity in computation and the requirement in control points. The first method needs considerable time to match the corresponding points. The second is ineffective when there are no three vanishing points. Therefore, in this paper, we propose a new camera calibration method that uses single monocular image by estimating the single vanishing point and width of the lane marker. It is not only greatly simplifies the computation, but also performs well in precision. This method was applied in the TuZhi car and the stable results suggest its promising future in the application of real time self-driving.

\section{Methodology}

\subsection{Lane Detection}

At first, we can detect the lane marker feature points in a 
perspective space and calculate edges of lane markers in image sequences. In order to determine which points belong to lane marker, the Hough transform is used to fitting the possible line segments of lane markers as the initial information of the lane markers. Then, we output the lines segments of the lane markers by iterating the initial information until it converges.

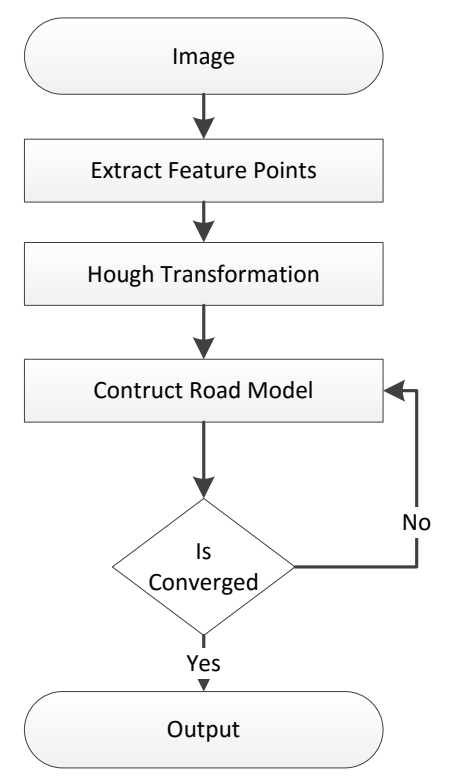

Fig 1. The process of lane detection

A scanning line is set to detect the row which contains lane markings in the image. The lane markings displayed in the image as a white line having a constant width. As the figure 2 shows, the black line means the gray value of pixels along the scanning line. In order to accurate locate edges of a lane marking, we propose a novel slider window with the length of $2 * \mathrm{~N}+1$ to process the image data. Because of the regularity of the gradient of the lane marker, the edge points of the lane marker will be appearing on the paired max and the min position with fixed width w.

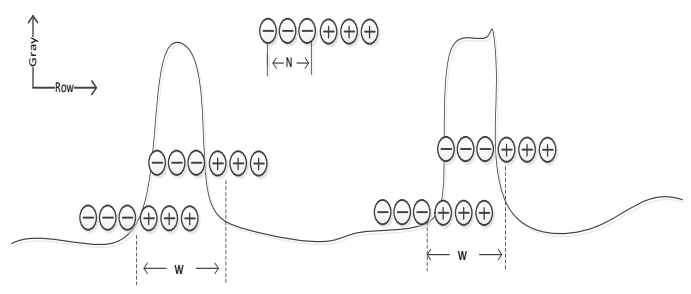

$$
\mathrm{g}(x)=\sum_{i=0}^{s} \sum_{k=-N}^{N} h(k) * f(i)
$$

Transform maps the image plane into the parameter space and count the line parameters. After the Hough Transformation, the lines are shown clearly in the image plane.

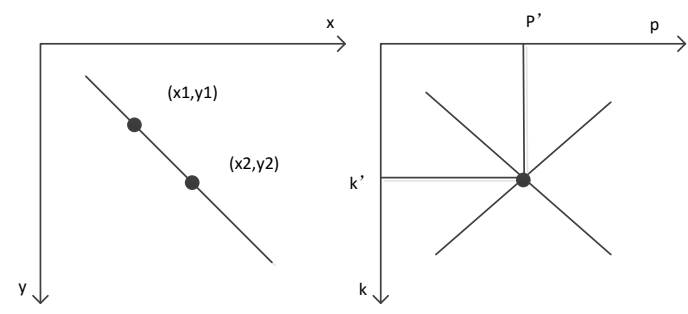

Fig 3. Hough Transform

Then we apply Hough Transform on the image. The Hough We make an information collector to store the information of lane marker to help our algorithm define which are true lane markers and how to choose them from possible line segments. By iterating, we can finally output the lane markers which have a high confidence level of $95 \%$.

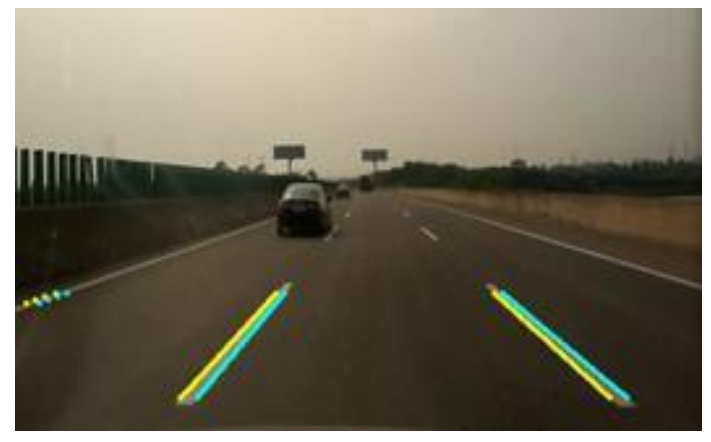

(a)

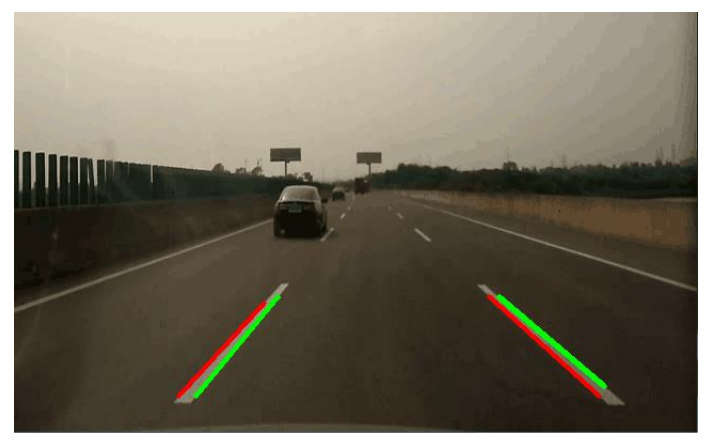

(b)

Fig 4. (a) edge points of lane markings. (b) line segment of lane markings

Fig2.The sliding window 


\subsection{Extraction of Vanishing Points}

A set of parallel lines in the real scenes which do not parallel to the image plane will be converted to a set of intersecting lines when projected to the image plane. And the vanishing points which contain the direction information of the lines are defined by the intersection points of the lines ${ }^{[10]}$. Finding the vanishing point greatly helps determine the estimation of attitude parameters of the camera since the relation between vanishing points and attitude parameters. In addition, in the road system, this process of analyzing will be greatly simplified by the lane marker detection.

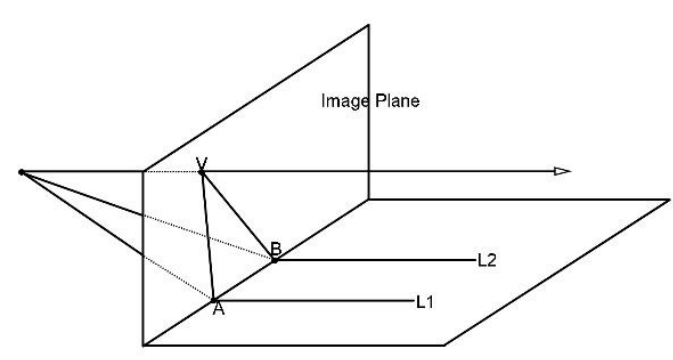

Fig 5. The diagram of vanishing point

After extracting the line segments of lane markers, we estimate the location of vanishing points based on the assumption that the lane markers coverage at the vanishing point. The tradition method is to calculate all the intersection points and choose the point where most line segments intersect. Once the number of line segments that we detect is large, this method is slow and lack of accuracy. Therefore, we put forward another method which is efficient in computation and of great accuracy, since such an inefficient method is not applicable in the real time detection.

As the solution, we separate the line segments according to their locations in the image into two set of lines, one is belonging to the left lane markers, another is belonging to right lane markers. During the computation of the intersection point, we only calculate the intersection points from different sets. We choose separately the line segment with a length threshold, mi mj from sets and calculate the intersection points weighted by the length of the line segment li lj involved in the calculation.
The location of the corresponding vanishing point hypothesis can be computed as ${ }^{[11]}$

$$
V_{\mathrm{ij}}=m_{i} \times m_{j}
$$

and the corresponding weight set to

$$
\mathrm{w}_{i j}=\left\|v_{i j}\right\| l_{i} l_{j}
$$

.And because of the high effectiveness of lane detection algorithm, the inaccuracy of the vanishing point is less than 2 pixels.

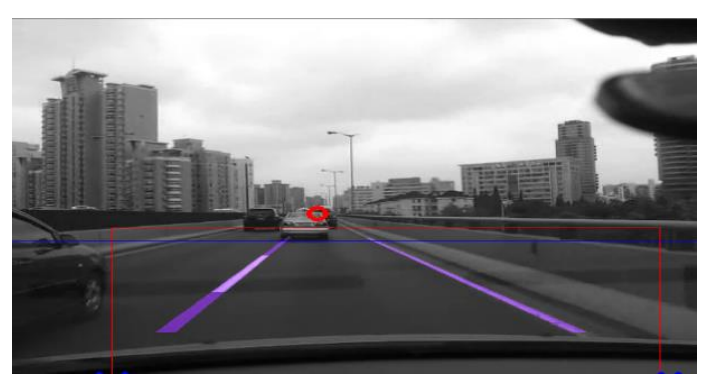

Fig 6. The center of the red circle is vanishing points

\subsection{Attitude Parameters Measuring}

In order to achieve a better result of attitude parameters, building a model revealing the relationship between the world coordinate and the image coordinate system is an essential part. What's more, the complexity of the model will greatly influence the process of the coordinate transform. Thus we create the model based on the position and pose of the camera. The figure 7 shows the model. The $\theta$ and $\gamma$ stand for the pitch angle and the course angel, $\mathrm{z}$ is the camera height.

Once the model is built, a 2D point can be projected into a 3D point by using the attitude parameters and the equalities. In other words, with the connection between the two coordinate system created, the $3 \mathrm{D}$ reconstruction of the road will be easily to complete.

According to the parameters mentioned above, the formula revealing the relationship between the two coordinate systems can be derived ${ }^{[12][13]}$ 


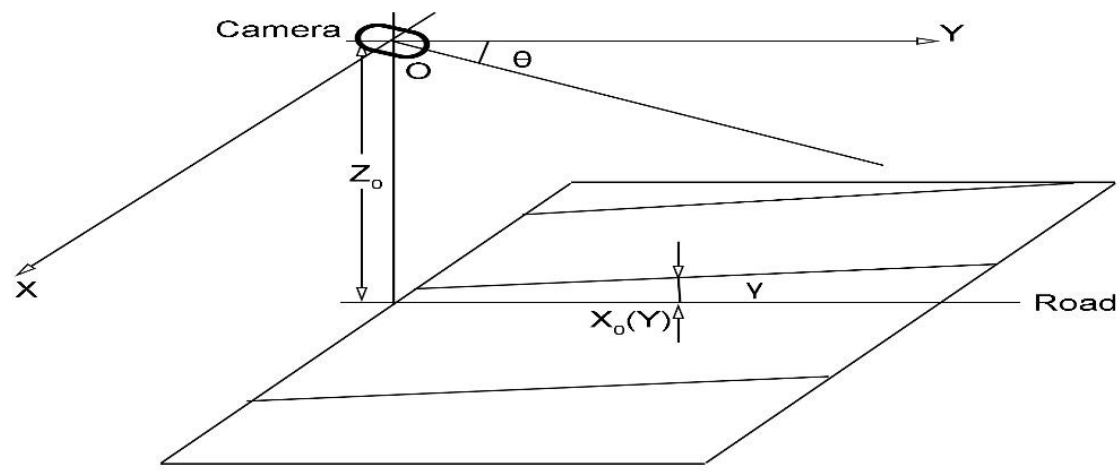

Fig 7. the camera and road model

$$
\begin{aligned}
& x(u, v)=h \times \cot \left(\theta-\alpha+u \times \frac{2 \alpha}{m-1}\right) \times \cos \left(\gamma-\beta+v \times \frac{2 \beta}{n-1}\right)+x_{0} \\
& y(u, v)=h \times \cot \left(\theta-\alpha+u \times \frac{2 \alpha}{m-1}\right) \times \sin \left(\gamma-\beta+v \times \frac{2 \beta}{n-1}\right)+y_{0}
\end{aligned}
$$

where $\quad \mathrm{u}, \mathrm{v}=$ image coordinate; $\mathrm{x}, \mathrm{y}, \mathrm{z}=$ space coordinate; $\theta=$ pitch angle; $\gamma=$ course angle; alpha=transverse angel of view; Longitudinal angle of view; $h=$ camera height $\mathrm{m}, \mathrm{n}=$ size of the image; $\mathrm{x} 0, \mathrm{y} 0, \mathrm{z} 0=$ camera's space coordinate.

From the formula above, another formula which transform the world coordinate into image coordinate can be derived

$$
\begin{gathered}
\mathrm{u}\left(\mathrm{x}, y, z_{0}\right)=\frac{\tan ^{-1}\left\{h \times \sin \left[\tan ^{-1}\left(\frac{y-y_{0}}{x-x_{0}}\right)\right] /\left(y-y_{0}\right)\right\}-(\theta-\alpha)}{2 \alpha / m-1} \\
v\left(\mathrm{x}, y, z_{0}\right)=\frac{\tan ^{-1}\left(\frac{y-y_{0}}{x-x_{0}}\right)-(\gamma-\beta)}{2 \beta / n-1}
\end{gathered}
$$

Thus we are creating the relationship between the two coordinate systems. According to the definition of

vanishing point, we can easily get the expression of it. In this part, we let $\mathrm{x}$ tend to infinity. The result is:

$$
\begin{aligned}
& \text { Vanish }_{u}=\lim _{x \rightarrow+\infty} u\left(x, y, z_{0}\right) \\
& \text { Vanish }_{v}=\lim _{x \rightarrow+\infty} v\left(x, y, z_{0}\right) \\
& \text { Vanish }_{u}=\frac{m-1}{2}-\frac{m-1}{2 \alpha} \theta \\
& \text { Vanish }_{v}=\frac{n-1}{2}-\frac{n-1}{2 \beta} \gamma
\end{aligned}
$$

So far,it is easily to find that the attitude parameters are highly related linear with the vanishing points. Therefore, it is very convenient to look up for the location of the vanishing point to compute the attitude parameters.

\subsection{Spatial Constraint to Solve Camera Height}

Once we get the attitude parameters, we can use the formula above or create the Rotation matrix to recover the connection between the real space and images. We get a point in the image and then transform it into the point in the real world or the scene.

For the camera height $h$, we put forward a new and highly effective approach to calculate it. Because of the width of lane marker and road lane is fixed under the standard structural road environment, we can automatically build a transformation matrix between perspective space and 3D space and solve the $h$ 
parameter.

For the edge points on the lane markers, we find the corresponding points by the regulations of parallel. By the least square fitting, not only can the parameter $h$ can be solved, but also the accuracy of attitude parameters can be greatly improved.

\section{Experiment}

We test our algorithm in the self-driving car 'TuZhi' (figure 8)with smart phone. The focal length is $1090 \mathrm{~mm}$,each frame's size is $1280 \times 720$. With the result of lane detection, the vanishing point with high accuracy can be calculated. According to the formula above, the attitude parameter and camera height $h$ can be estimated. Thus, we apply inverse perspective transformation on the image to reconstruct the road. With a set of continuous images, the road can be stitched. In addition, the width of the road or the distance between cars can be estimated accurately. From figure 9, the results of the 3D reconstruction are clearly shown. All the roads and lane markers become a set of parallels again.
Strictly speaking, because of the existence of other vanishing points, the distance in the real world of the selected points are not equal to the lane width. But we can prove that this error is less than 2 pixels which is a very small error that can be negligible under the condition of $\gamma<5^{\circ}$.

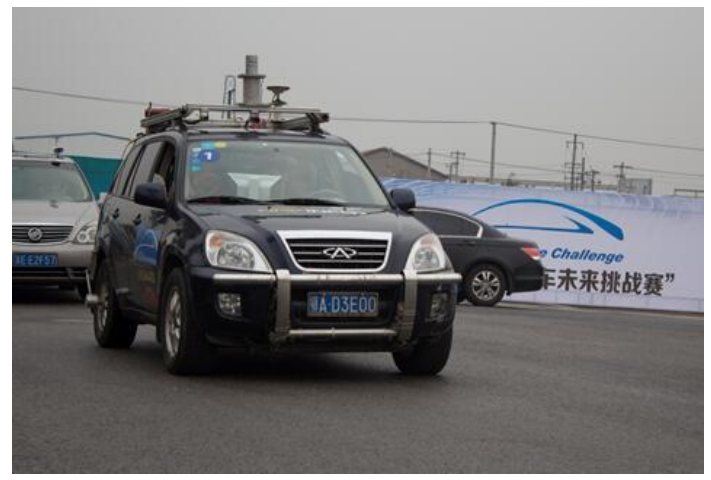

Fig 8.’TuZhi’ self-driving car.
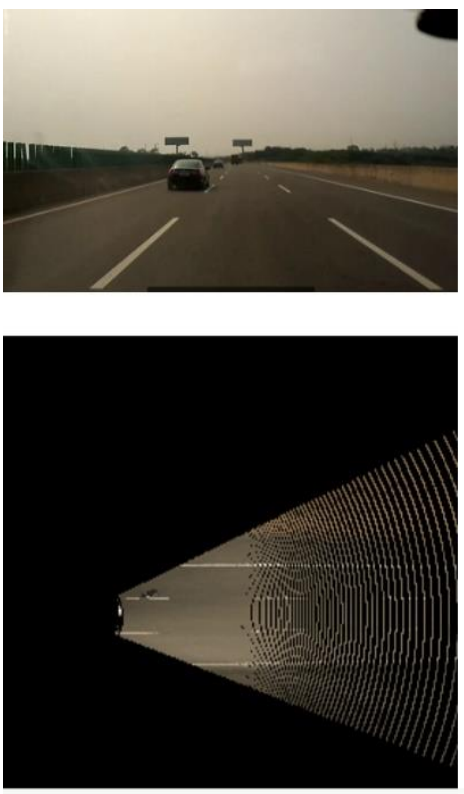

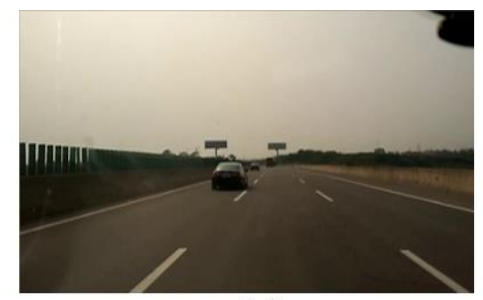

(a)

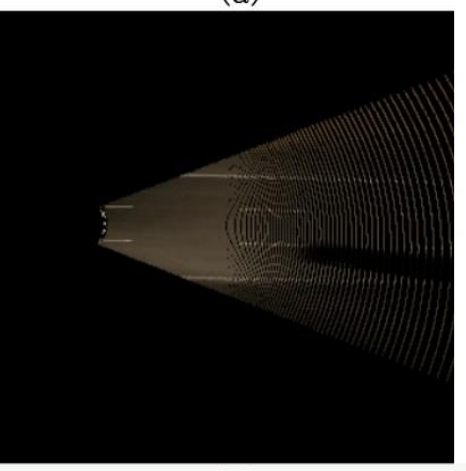

(b)
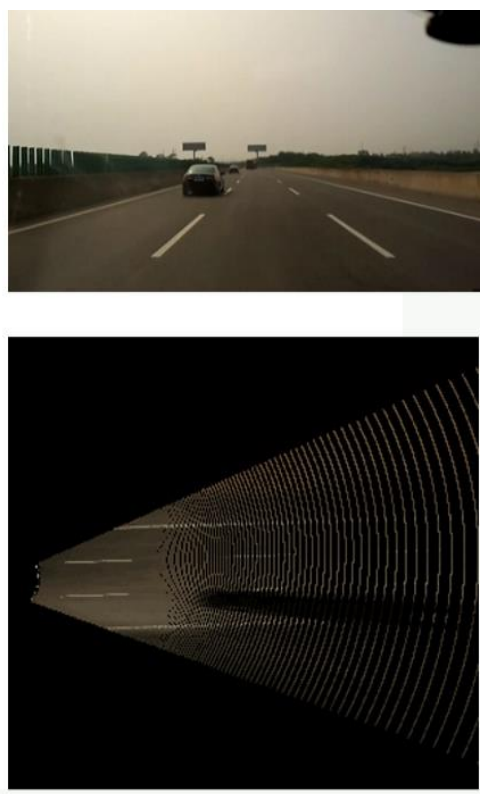

Fig 8. Experiment result, figure $\mathrm{a}$ is the original image, figure $\mathrm{b}$ is the result of 3D reconstruction

\section{Conclusion}

We have proposed a simple and real-time approach for the camera calibration, based on the vanishing points and the geometrical constraint of the lane marker width. By using the images from smart phone installed on the 'TuZhi' car, the result is accurate enough for many applications. As its simplification in computation, it can be widely used in self-driving car. 
However, this method's precision is closely related to the improvement in the work.

\section{Acknowledge}

I would like personally thank the following people and institutes which support me a lot with my gratitude.

At first, without the help of my tutor BiJun Lee,I would not complete my experiment successfully. It is the kind encouragement and massive instructions that benefit me a lot.

Then, I wish to express my gratitude to the funding of the program, Chinese National Natural Science Foundation. With their great support, I can devote myself to the experiment and research.

Finally, I want to thank all the people in the laboratory who supplied me a lot of data of great value.

\section{REFERENR}

[1] Bas, E.K., Crisman, J.D., 1997. An easy to install camera calibration for traffic monitoring. Intelligent Transportation System, 1997. ITSC '97., IEEE Conference on, pp. 362-366. G. S. K. Fung, N. H. C. Yung, G. K. H. Pang,2003. Camera calibration from lane marker. Optical Engineering,43, pp.2967-2977.

[2] G. S. K. Fung, N. H. C. Yung, G. K. H. Pang,2003.Camera calibration from lane marker. Optical Engineering,43,pp.2967-2977

[3] Hattori, H., 2000. Stereo for 2D visual navigation. In telligent Vehicles Symposium, 2000. IV 2000. Proceed ings of the IEEE, pp. 31-38.

[4] Bishop, R., 2000. A survey of intelligent vehicle applications worldwide. Intelligent Vehicles Symposium, accuracy of detection of vanishing point. It still has some

2000. IV 2000. Proceedings of the IEEE. IEEE, pp. 25-30.

[5] Yuan, S., Zheng, T., Liru, D., 2005. Camera SelfCalibration and Three Dimensional Reconstruction Using Spatial Orthogonal Constraints [J]. Journal of Xi'an Jiaotong University 2, 007.

[6] Liang, D., Wu, F.-c., Ruan, Z.-c., WEI, S., 2002. Determining Camera Intrinsic Parameters and Pose from 5 Control Points. Journal of University of Science and Technology of China 32, 194-201.

[7] Han Long, Wang Zeng-fu,2008. High precision feature detection and camera calibration based on geometrical constraint. Journal of University of Science and Technology of China,38(10), pp.1211-1217.

[8] XIE, W.-h., ZHANG, Z.-x., ZHANG, J.-q., 2003. New method of camera calibration with vanishing points $[\mathrm{J}]$. Journal of Harbin Institute of Technology 11, 033.

[9] QIU, J., LIU, Z., YANG, R., 2005. Vanishing Points Based Modeling from a Single Image. Computer Engineering 22, 069.

[10] Yu, C., Ying, F., Yuntao, Y., 2011. Method of attitude angle measuring based on disappear point lookup in 3D reconstruction of road surface. Chinese Journal of Sensors and Actuators 24, 1081-1086.

[11] Szeliski, R., 2010. Computer vision: algorithms and applications Lines. pp. 255

[12] Bertozzi, M., Broggi, A., 1998. GOLD: A parallel realtime stereo vision system for generic obstacle and lane detection. Image Processing, IEEE Transactions on 7, 6281.

[13] Guo, L., XU, Y.-c., LI, K.-q., LIAN, X.-m., 2006. Study on real-time distance detection based on monocular vision technique. Journal of Image and Graphics 11, 74-81. 